\title{
WT1 Peptide-loaded Allogeneic Dendritic Cell Vaccine
}

National Cancer Institute

\section{Source}

National Cancer Institute. WT1 Peptide-loaded Allogeneic Dendritic Cell Vaccine. NCI

Thesaurus. Code C120185.

A cell-based cancer vaccine composed of donor-derived dendritic cells (DCs) loaded with three, human leukocyte antigen A2 (HLA-A2)-binding peptides derived from the human tumor-associated antigen (TAA) Wilms tumor protein 1 (WT1), with potential immunomodulating and antineoplastic activities. Upon vaccination, WT 1 peptide-loaded allogeneic DC vaccine exposes the immune system to WT1-derived peptides and may stimulate the host immune system to mount a cytotoxic T-lymphocyte (CTL) response against WT 1-expressing cancer cells, which could result in tumor cell lysis. WT 1, a transcription factor, is overexpressed in leukemic cells and in various solid tumors, and only minimally in normal tissues; its expression often correlates with disease progression and poor prognosis. HLA-A2 is an MHC class I molecule that presents antigenic peptides to CD8+ T-cells; restricting the peptides to those epitopes that bind most efficiently to HLA-A2 may improve antigenic peptide immunogenicity. 